\title{
An empirical investigation of the laser survivability curve: $\mathrm{V}$
}

Jonathan Arenberg, Wolfgang Riede, Allesandra Ciapponi, Paul Allenspacher, Jon Herringer, et al.

Jonathan Arenberg, Wolfgang Riede, Allesandra Ciapponi, Paul Allenspacher, Jon Herringer, Denny Wernham, "An empirical investigation of the laser survivability curve: V," Proc. SPIE 9237, Laser-Induced Damage in Optical Materials: 2014, 923723 (31 October 2014); doi: 10.1117/12.2068257

SPIE. Event: SPIE Laser Damage, 2014, Boulder, Colorado, United States 


\title{
An empirical investigation of the laser survivability curve: $\mathbf{V}$
}

\author{
Jonathan Arenberg ${ }^{1}$, Wolfgang Riede ${ }^{2}$, Alessandra Ciapponi ${ }^{4}$, Paul Allenspacher $^{2}$, Jon Herringer ${ }^{3}$ \\ and Denny Wernham ${ }^{4}$
}

(1) Northrop Grumman Aerospace Systems, One Space Park Drive, Redondo Beach CA 90278, USA

${ }^{(2)}$ German Aerospace Center (DLR), Institute of Technical Physics, Stuttgart, Germany

(3) Arrow Thin Films, Inc., Wyckoff, NJ, USA

${ }^{(4)}$ European Space Agency, ESTEC, Nordwijk, Netherlands

\begin{abstract}
In this paper, we report on a continuing multi-year empirical investigation into the nature of the laser survivability curve. The laser survivability curve is the onset threshold as a function of shot number. This empirical investigation is motivated by the desire to design a universal procedure for the measurement of the so-called S on 1 damage threshold. In this year's paper we investigate the usefulness of scaling the fluence with shot number. First the scaling process is defined and applied to a result from our experimental archives. The probability of damage curve for a single shot test is extrapolated to $10^{4}$ shots. The scaled result is shown to be very close the observed results providing a basis for extrapolation to very large values of $n$.
\end{abstract}

Keywords: Laser damage testing, laser optics qualification, S on 1 testing, ISO 11254-2, ISO 21254-2

\section{Introduction}

In this poster, we report on a continuing empirical investigation into the nature of the laser survivability curve. $[1,2,3,4]$ In this year's report, we investigate a new method for processing data from $S$ on 1 testing. Last year, we discussed a maximum likelihood method, using a binomial based probability model. [4] Last year's results seemed to produce very conservative estimated of the safe operating fluence for large shot numbers.

\section{Scaling Procedure}

In this year's investigation into the form of $P(\phi, n)$ a "remapping" of the effective fluence is used to extrapolate to large values of shot numbers.

For this work, we assume a Weibull (two parameter) as the form of the damage probability curve

$$
\mathrm{P}(\phi)=1-e^{-\left(\frac{\phi}{\eta}\right)^{\beta}}
$$

Equation (1) is rewritten to explicitly apply to the case of $n$ shots with fluence scaling as 


$$
\mathrm{P}(\phi, n)=1-e^{-\left(\frac{\phi r(n)}{\eta}\right)^{\beta}}
$$

The term fluence scaling is derived from the definition of $\phi r(n)$ as a scaled representation of the true fluence $\phi$.

The process for the determination of the scaling is:

1. Determine the probability of damage for $n=1, P(\phi, 1)$

2. Determine the probability of damage for $n=10,000, P\left(\phi, 10^{4}\right)$

3. Using (2) to determine the scaling factor $r$ between fluences for the same $P$ and different $n$

We used the data from a test performed at DLR earlier in our project. This sample is a conventionally manufactured AR coating designed for $1064 \mathrm{~nm}$. The test was conducted at $1064 \mathrm{~nm}$, with a pulse width of $3.5 \mathrm{~ns}$ and a spot size of $400 \mu \mathrm{m}$ mean diameter $1 / \mathrm{e}^{2}$ and a $100 \mathrm{~Hz}$ pulse repetition frequency. The test procedure followed the ISO standard for S on 1 testing, ISO 21254 Part 3.[5]

Figure 1 shows the test results for $\mathrm{n} 1$ and the corresponding probability of damage curve, $\mathrm{P}(\phi, 1)$. The $S$ on 1 data was segregated for data where $n=1$ and a maximum likelihood method used to determine Weibull model parameters called for by Step 1. [6] The values determined for $n=1$, were $\eta_{1}=9.8 \mathrm{~J} / \mathrm{cm}^{2}$ and $\beta_{1}=6.5$. Figure 2 shows the test results for $n^{\sim} 10^{4}$ and the corresponding Weibull model. The values determined for $n=10^{4}$, were $\eta_{10 k}=8.5 \mathrm{~J} / \mathrm{cm}^{2}$ and $\beta_{10 k}=6.4$.

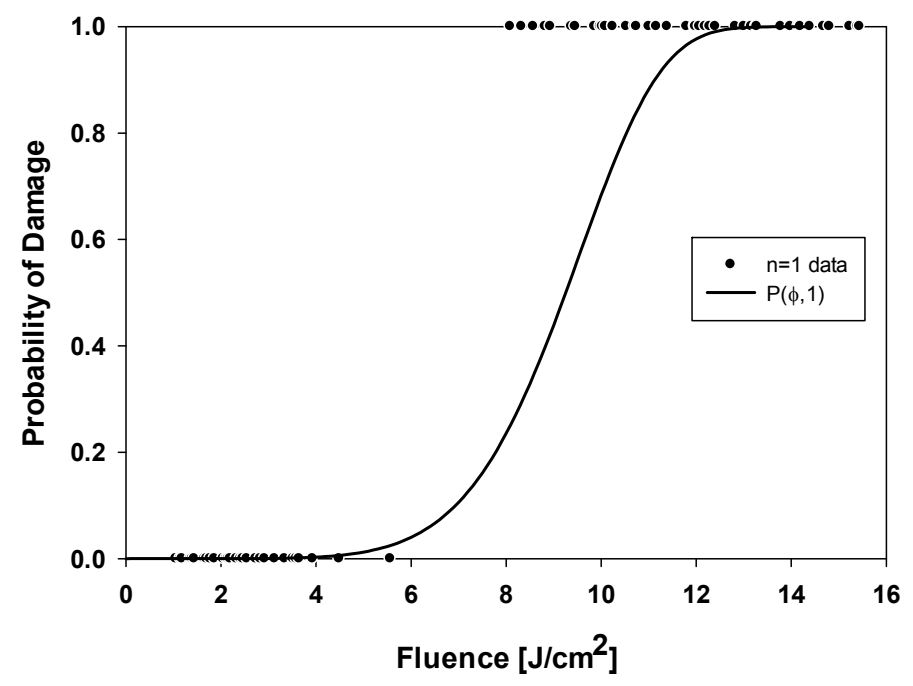

Figure 1 


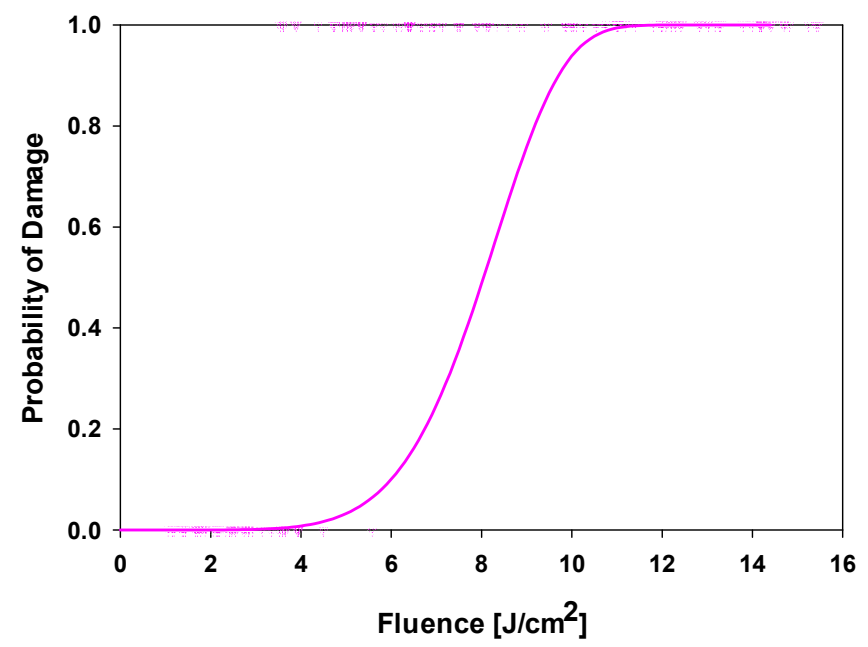

Figure 2

To complete Step 2, $r(n)$ must be determined. An arbitrary value of $P, 0.7$, is selected and (2) along with the proper parameters for $n=1$ and $n=10^{4}$ are used to determine $\phi_{1}$ and $\phi_{10 k}$. Figure 3 shows this graphically, the curves for $\mathrm{P}(\phi, 1)$ and $\mathrm{P}\left(\phi, 10^{4}\right)$ are shown and the horizontal line is $\mathrm{P}=0.7$

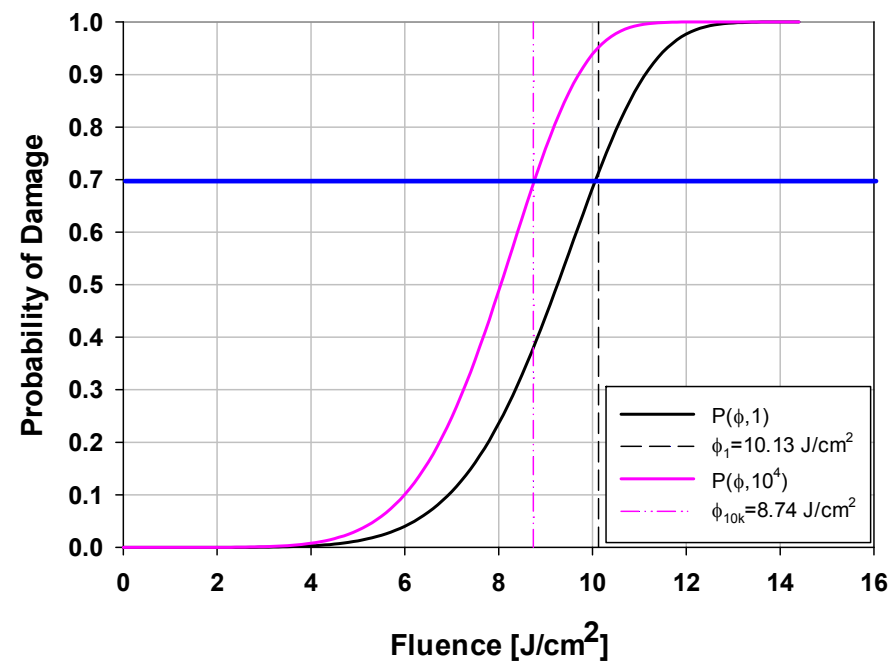

Figure 3

Using (2) we can write

$$
\mathrm{P}\left(\phi_{1}, 1\right)=\mathrm{P}\left(\phi_{10 k}, 10^{4}\right)
$$


So

$$
1-e^{-\left(\frac{\phi_{1}}{\eta_{1}}\right)^{\beta_{1}}}=1-e^{-\left(\frac{\phi_{10 k} k(n)}{\eta_{10 k}}\right)^{\beta_{10 k}}}
$$

Subtracting 1 from each side of (4) and equating exponents gives

$$
\left(\frac{\phi_{1}}{\eta_{1}}\right)^{\beta_{1}}=\left(\frac{r(10 k) \phi_{10 k}}{\eta_{10 k}}\right)^{\beta_{10 k}} .
$$

We chose a form for $r(n)$ suggested by the theory of mechanical fatigue, $r(n)=n^{\alpha}$.[6] It should be noted, that $n^{\alpha}$ has the handy property that $r(1)=1$ for all $\alpha$.

Solving (5) for $r(10 k)$ gives

$$
r(10 k)=\frac{\eta_{10 k}}{\phi_{10 k}}\left(\frac{\phi_{1}}{\eta_{1}}\right)^{\beta_{1} / \beta_{10 k}}
$$

Since $r$ has the form $r=n^{\alpha}$

$$
n^{\alpha}=\frac{\eta_{10 k}}{\phi_{10 k}}\left(\frac{\phi_{1}}{\eta_{1}}\right)^{\beta_{1} / \beta_{10 k}}
$$

Taking the logarithm of both sides of (7) and solving for a gives

$$
\alpha=\frac{\ln \left(\frac{\eta_{10 k}}{\phi_{10 k}}\left(\frac{\phi_{1}}{\eta_{1}}\right)^{\beta_{1} / \beta_{10 k}}\right)}{\ln (n)}
$$

So since $n=10,000, \alpha$ is given

$$
\alpha=\frac{\ln \left(\frac{\eta_{10 k}}{\phi_{10 k}}\left(\frac{\phi_{1}}{\eta_{1}}\right)^{\beta_{1} / \beta_{10 k}}\right)}{\ln (10,000)} .
$$

Evaluation of (9) gives, $\alpha=0.0152$. The scaled (from $n=1$ values) is given

$$
P(n, \phi)=1-e^{-\left(\frac{\phi n^{\alpha}}{\eta_{1}}\right)^{\beta_{1}}} .
$$


Figure 4 shows excellent agreement between the scaled curve, (10) and the measured curve for $n=10^{4}$. There are three traces on Figure $4, \mathrm{P}(\phi, 1), \mathrm{P}\left(\phi, 10^{4}\right)$ and the scaled result from (10). There appear to be only two, since $\mathrm{P}\left(\phi, 10^{4}\right)$ and $(10)$ are nearly identical, a truly surprising and amazing result.

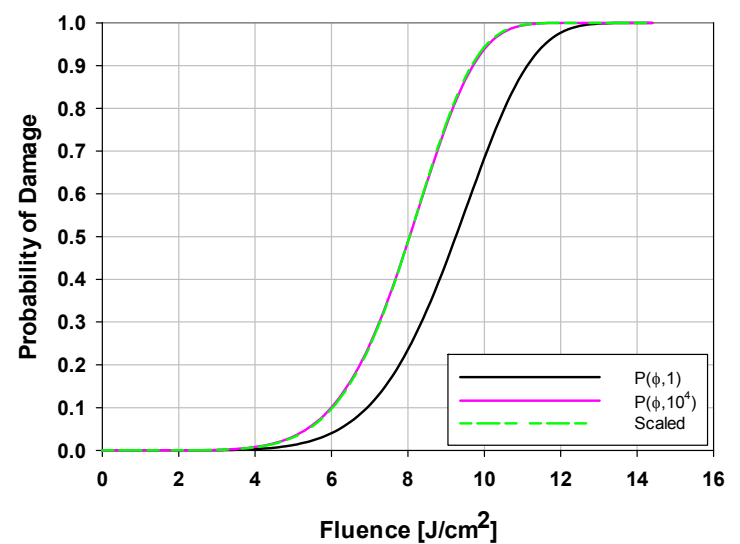

Figure 4

Generalizing to other $n$ values, via manipulation of (10) gives a general expression for the evolution of the $S$ on 1 threshold for various probabilities of damage (sometime before $t^{t h e} n^{\text {th }}$ shot) entirely in terms of the scaled result

$$
\phi(n, P)=\frac{\eta_{1}}{n^{\alpha}} \exp \left[\frac{1}{\beta_{1}} \ln \left(\ln \left(\frac{1}{1-P}\right)\right)\right] .
$$

Figure (5) shows the plot for the safe operating fluence for an assumed risk (probability of damage, P) and shot number. Note, that in contrast with previous predictions, there is no arbitrary cliff, in contrast with last year's results. [4]

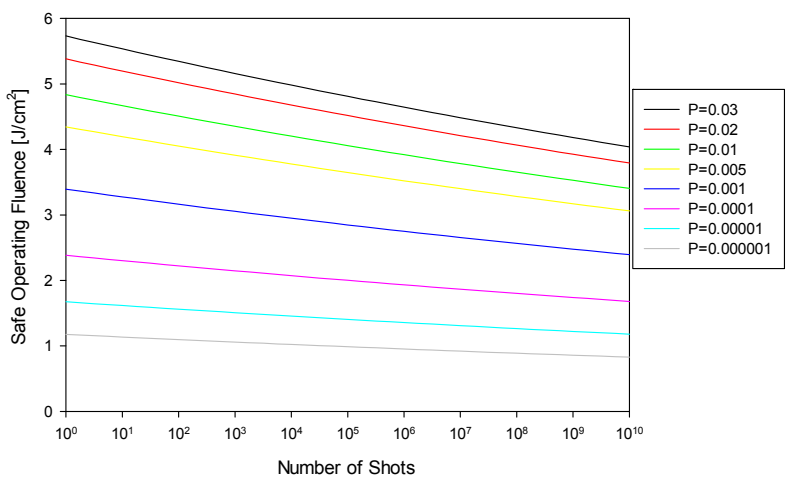

Figure 5 


\section{Summary \& Next Steps}

The method of scaling fluence produces smooth and at first viewing reasonable predictions. In order to validate the generalizability of these results we must apply this method across our data set, and assure ourselves that the method is stable. Watch for our results in 2015 , we look forward to sharing them with you.

\section{References}

[1] Arenberg, J.W.,Riede, W., Ciapponi, A., Allenspacher, P. and Herringer, J., "An empirical investigation of the laser survivability curve",Proc. SPIE 7842, Laser-Induced Damage in Optical Materials: 2010, 78421B (2 December 2010); doi: 10.1117/12.867349

[2] Arenberg, J.W.,Riede, W., Ciapponi, A., Allenspacher, P. and Herringer, J., "An empirical investigation of the laser survivability curve:II", SPIE 8190, Laser-Induced Damage in Optical Materials: 2011, 819019 (23 November 2011); doi: 10.1117/12.899271

[3] Herringer, J.,Riede, W., Ciapponi, A., Allenspacher, P. and ] Arenberg, J.W., "An empirical investigation of the laser survivability curve:II", Proc. SPIE 8530, Laser-Induced Damage in Optical Materials: 2012, 85301N (4 December 2012); doi: 10.1117/12.977477

[4] Arenberg, J.W.,Riede, W., Ciapponi, A., Allenspacher, P., Herringer, J. and Wernham, D., “An empirical investigation of the laser survivability curve:IV", Proc. SPIE 8885, Laser-Induced Damage in Optical Materials: 2013, 88851P (November 15, 2013); doi:10.1117/12.2030390

[5] Arenberg, J.W. and Thomas, M.D., "Laser damage threshold measurements via maximum likelihood estimation" ,Proc. SPIE 8885, Laser-Induced Damage in Optical Materials: 2013, 888500 (14 November 2013); doi: 10.1117/12.2030259

[6] Beer, Ferdinand P, and E. Russell Johnston, Jr. Mechanics of Materials. 2nd ed. New York: McGrawHill, Inc. 1992. 